\title{
Extremeness Aversion and Attribute-Balance Effects in Choice
}

\author{
ALEXANDER CHERNEV*
}

\begin{abstract}
Consumers often make decisions based on the extremeness of choice alternatives. Prior research has argued that extremeness aversion is a function of the relational properties of choice alternatives and that the middle option, defined such that its attribute values are between the values of the other alternatives, is always viewed as the least extreme, compromise option. The attribute-balance hypothesis advanced in this article extends prior research, demonstrating that extremeness aversion is also a function of the dispersion of attribute values within each alternative. In particular, it is argued that an option with equal attribute ratings will be perceived as the compromise alternative even when it is not the middle option. Data from three studies support this prediction, offering converging evidence for the attributebalance effect in different decision contexts. Theoretical implications of the findings are discussed in the context of the extant extremeness aversion research.
\end{abstract}

$\mathrm{O}$ ne of the major findings that has emerged from recent decision research is the presence of extremeness aversion in choice. Extremeness aversion extends the principle of loss aversion (Kahneman, Knetsch, and Thaler 1991; Tversky and Kahneman 1991) to advantages and disadvantages that are defined relative to the other options in the choice set, rather than relative to a readily available reference point. In this context, extremeness aversion refers to the finding that, all else being equal, an option with relatively more extreme values tends to be viewed as less attractive than an otherwise equivalent option with moderate values.

Extremeness aversion, although intuitive, is counter to the assumption of independence of irrelevant alternatives, which states that the preference between options does not depend on the presence or absence of other options and, hence, that a new alternative added to a set takes its share from existing options in proportion to their original shares (Luce 1959). Extremeness aversion is also counter to the principle of betweenness inequality (Simonson and Tversky 1992) and the substitution effect (Huber and Puto 1983; Tversky 1972), according to which adding an adjacent nondominated alternative should draw larger share from the middle (more similar) alternative than from the extreme (less similar) alternative. Thus, extremeness aversion implies that adding an adjacent nondominated alternative will draw a larger share from the extreme rather than from the middle alter-

*Alexander Chernev is associate professor of marketing, Kellogg School of Management, Northwestern University, 2001 Sheridan Road, Evanston, IL 60208 (ach@northwestern.edu). The author thanks the editor, the associate editor, and the reviewers for their constructive comments. This research was partially funded by the Kraft Research Chair, received by the author. native. To illustrate, consider a set of two-dimensional alternatives $\{x, y, z\}$, where $y$ is between $x$ and $z$ (e.g., $x_{1}<$ $y_{1}<z_{1}$ and $x_{2}>y_{2}>z_{2}$, as shown in fig. 1). In this context, the extremeness aversion principle predicts that because $y$ has small advantages and disadvantages with respect to $x$ and $z$, whereas both $x$ and $z$ have larger advantages and disadvantages with respect to each other, $y$ will be more preferred in the choice set $\{x, y, z\}$ than in either of the sets $\{x, y\}$ and $\{y, z\}$.

Two forms of extremeness aversion are commonly considered in the literature: compromise and polarization. Compromise (Simonson 1989; see also Dhar, Nowlis, and Sherman 2000; Kivetz, Netzer, and Srinivasan [2004]; and Wernerfelt 1995) occurs when the extremeness aversion is symmetric with respect to both attributes describing choice alternatives. Thus, option $y$ is perceived to be more attractive when evaluated in the context of a triple $\{x, y, z\}$ than in both pairs $\{y, x\}$ and $\{y, z\}$. In contrast, polarization occurs when the extremeness aversion is asymmetric: option $y$ is perceived to be more attractive when evaluated in a triple $\{x, y, z\}$ than in one of the two pairs $\{y, x\}$ or $\{y, z\}$ - but not in both. The asymmetric pattern of extremeness aversion observed in the polarization effect has been attributed to the asymmetric pattern of the relative importance of the attributes describing choice alternatives (Simonson and Tversky 1992). Assuming the attributes are equally important, however, both effects should lead to the same prediction, whereby adding an extreme alternative $x$ or $z$ is likely to increase the choice share of the middle alternative $y$.

A cornerstone assumption of the extremeness-aversion principle is that decision makers rely on the choice context to determine the extremity of each alternative, such that advantages and disadvantages of each alternative are defined 
FIGURE 1

EXTREMENESS AVERSION IN CHOICE

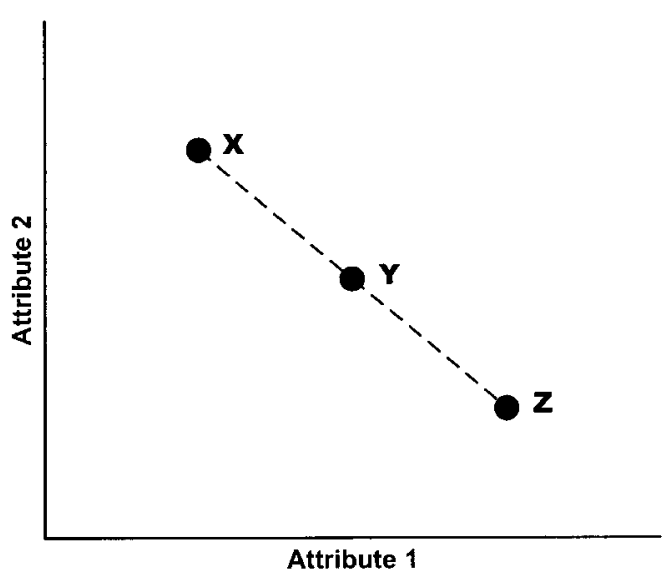

relative to one another. Therefore, the extremity of any given option can be defined in the context of at least three options; there is no extremeness aversion in binary choice because no option is more extreme than another. In this context it is assumed that the middle option, defined such that its attribute values are between the values of the other alternatives (e.g., option $y$ in fig. 1), is always the option that becomes the compromise choice (Tversky and Simonson 1993). The assumption that the middle option becomes the compromise is also very intuitive because the values of the middle alternative on each of the attributes lie between the values of the other, more extreme options.

Research presented in this article questions the assumption that extremeness aversion is based only on the relational properties of the choice alternatives. It is argued that, in certain cases, individuals can use the available attribute ratings to construct reference points that are, in turn, used to evaluate the extremity of the choice alternatives. In this context, it is proposed that the middle option is not always the compromise alternative, and, in some cases, that one of the adjacent options ( $x$ and $z$ in fig. 1) can actually become the compromise. The viability of this proposition is tested by identifying a scenario in which individuals' preferences are affected not only by the decision context, defined by the relational properties of choice alternatives, but also by alternative-specific factors that are relatively independent from the decision context. This scenario is illustrated by introducing the notion of scale equivalence and discussing how the presence of balanced alternatives might influence extremeness aversion in choice.

\section{SCALE EQUIVALENCE AND ATTRIBUTE BALANCE IN CHOICE}

The proliferation of "smart agents" and online product comparison tools has spearheaded a trend toward standardization of the metrics representing overall product performance. To illustrate, Consumer Reports, BizRate, and Mor- ningstar are using aggregate product ratings such as quality, reliability, and performance. The use of these ratings has been driven to a large degree by the need for comparability across product categories, as well as by an attempt to simplify the increasing number and complexity of product attributes.

Using aggregate ratings of product performance, such as ease of use, confidence, customer service, cost, reliability, and variety has also led to using similar rating scales to describe product performance on different dimensions. $\mathrm{Nu}-$ meric rating scales, stars, circles, and even smiley faces are commonly used to rate stocks, mutual funds, financial services, and consumer electronics. The use of scales with similar metrics raises the question of how consumers evaluate choice alternatives in cases where attribute ratings are directly comparable, and, specifically, how consumers evaluate alternatives with equal attribute values. To illustrate, consider a choice alternative described on two attributes using the same 100-point rating scale, such that the alternative has equal ratings on both attributes, say $(60,60)$. This alternative is referred to as balanced, and it is proposed that attribute balance can fundamentally change consumer evaluations of the extremeness of the options in the choice set.

This research posits that the use of attribute scales sharing the same metric (e.g., 100-point scales) makes the comparison of attribute values describing each alternative more salient than the comparison of options' values on each attribute. In the case of balanced alternatives, this effect is compounded by the fact that these alternatives have identical attribute values and, hence, are more likely to be directly compared in choice-an argument that implies a psychological mechanism similar to the processes that underlie the Gestalt principle of grouping by similarity (Baylis and Driver 1992; Quinlan and Wilton 1998; Wertheimer [1923] 1999). Building on the notion of similarity assessment as a core decision heuristic, this research argues that, by virtue of having equal attribute ratings, the balanced option becomes a reference point in evaluating the extremeness of choice alternatives. The analysis of how attribute balance influences extremeness aversion and choice is outlined in the next section.

\section{EXPERIMENTAL HYPOTHESES}

Consider a choice set composed of four alternatives, each described on two attributes using the same 100-point rating scale: A $(50,70), \mathrm{B}(60,60), \mathrm{C}(70,50)$, and D $(80,40)$, as shown in figure 2. Prior extremeness research predicts that, because the values of choice alternatives can be ordered so that $a_{1}<b_{1}<c_{1}<d_{1}$ and $a_{2}>b_{2}>c_{2}>d_{2}$, adding option $\mathrm{A}$ to the set $\mathrm{BC}$ will make option $\mathrm{B}$ a compromise and will increase its relative share, whereas the addition of option $\mathrm{D}$ to the set $\mathrm{BC}$ should make option $\mathrm{C}$ a compromise and increase its relative share.

Research presented in this article argues that the above predictions are contingent upon the scale-equivalence properties of choice alternatives. In particular, it is proposed that attribute balance is likely to make option B the least extreme 
FIGURE 2

EXTREMENESS AVERSION AMD ATTRIBUTE BALANCE

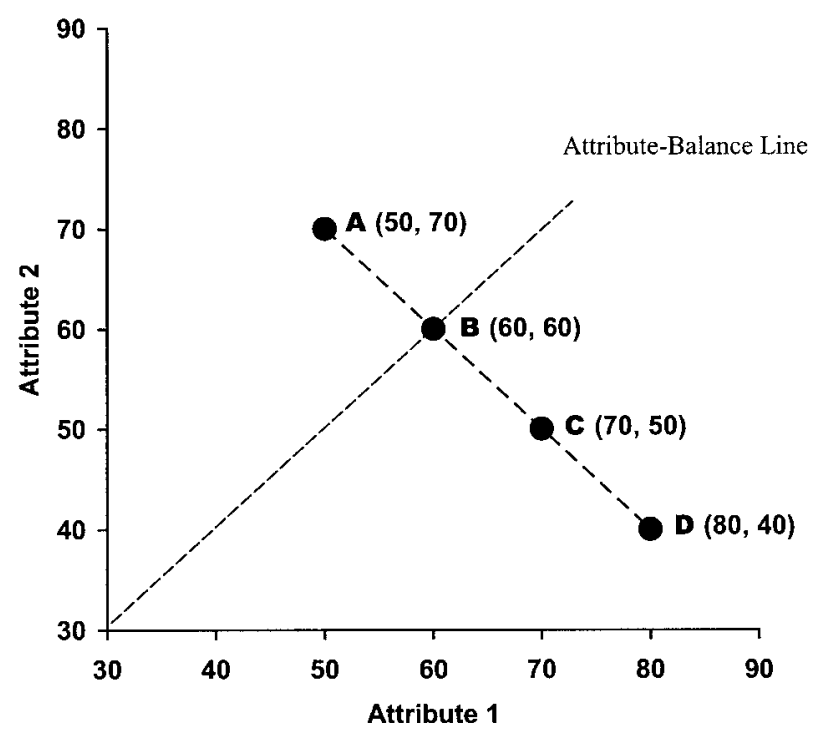

NotE.-Attribute values are given in parentheses. Option B $(60,60)$ is the balanced alternative.

option regardless of its relative position in the choice set. This argument leads to the counterintuitive prediction that in the set $\mathrm{BCD}$, the compromise alternative is not option $\mathrm{C}$, as predicted by prior research, but the balanced option $\mathrm{B}$.

Why would consumers perceive the balanced option rather than the middle option to be the compromise alternative? When the attributes describing choice alternatives use the same metric, individuals can readily compare an option's performance on different attributes. In this context, an option's extremeness can be evaluated not only based on its relative position in the choice set but also based on the proximity of its own attribute values. Thus, when the extremeness of a balanced alternative is evaluated based on the proximity of its own attribute values, this option is likely to be selected as the least extreme, compromise alternative. In contrast, when an option's attributes are not directly comparable and alternatives are evaluated relative to one another, the middle option is likely to be selected as the compromise option.

To illustrate, consider option B (fig. 2), which lies on the attribute-balance line-a hypothetical line composed of all potential options with identical values on both attributes. It is proposed that, when evaluating options' extremeness, consumers rely not only on the distance between options' values relative to one other (i.e., along the $\mathrm{AD}$ line) but also on their distance from the attribute-balance line. The farther away an option is from the attribute-balance line, the greater is its perceived extremeness. It is therefore proposed that the balanced option B will be perceived as the compromise option in the set BCD, which decreases the likelihood that the middle option will be perceived as the compromise al- ternative. In contrast, when the middle option is also balanced, the likelihood increases that this option will be perceived as the compromise choice (compared to a set in which neither of the options is balanced). These predictions can be more formally summarized as follows:

H1: The selection of the compromise option from a trinary set is a function of the attribute balance of the alternatives, as follows:

H1a: The middle option is more likely to be perceived as the compromise in sets without a balanced alternative than in sets with an adjacent (nonmiddle) balanced alternative $(\{x, y, z\}$ vs. $\{x, y$, $\left.\left.z_{B}\right\}\right)$.

H1b: The middle option is more likely to be perceived as the compromise when this option is balanced than when none of the options is balanced $(\{x$, $y, z\}$ vs. $\left.\left\{x, y_{B}, z\right\}\right)$.

The attribute-balance view of extremeness aversion also has important implications for choices made from binary sets. Because the attribute-balance effects are independent of the relational properties of the choice alternatives, extremeness aversion should also be observed in binary choice. To illustrate, an option with attribute values $(70,50)$ is likely to be perceived as more extreme than an option with attribute values $(60,60)$, which, in turn, is more likely to be perceived as the compromise option. This proposition is a departure from the relational approach, which implies that there is no extremeness aversion in binary choice because the binary nature of the decision set does not allow individuals to create the relational reference point necessary to assess an option's extremeness. The attribute-balance predictions on extremeness aversion in binary choice sets can be formally expressed as follows:

H2: The selection of the compromise option from a binary set is a function of attribute balance, such that the balanced alternative is likely to be perceived as the compromise option.

Based on the above discussion, further predictions can be made about the impact of attribute balance on the compromise effect. Recall that the compromise effect predicts that adding an adjacent alternative to a binary set is likely to lead to an increase in the relative share of the middle alternative. The above discussion suggests that the compromise effect should also be a function of the attribute balance of choice alternatives. In particular, based on the presence and the relative position of the balanced alternative in the core (binary) and the extended (trinary) sets, four scenarios for testing the attribute-balance effect are identified: (1) a balanced alternative is added to a set in which none of the options is balanced: $\{x, y\} \rightarrow\left\{x, y, z_{B}\right\}$; (2) one of the options in the core set is balanced and it becomes one of the adjacent options in the extended set, $\left\{x, y_{B}\right\} \rightarrow\left\{z, x, y_{B}\right\}$; (3) one of the options in the core set is balanced and it becomes the 
middle option in the extended set, $\left\{x, y_{B}\right\} \rightarrow\left\{x, y_{B}, z\right\}$; and (4) none of the options in the core or extended set is balanced, $\{x, y\} \rightarrow\{x, y, z\}$. The last scenario is used as a benchmark to evaluate the impact of attribute balance on choice.

The first scenario raises the question of how adding an adjacent balanced alternative to the core set (i.e., $\{x, y\} \rightarrow$ $\left.\left\{x, y, z_{B}\right\}\right)$ is likely to affect the compromise effect. Prior research has shown that, in the absence of balanced alternatives, the relative share of the middle option is likely to increase because it becomes the compromise option in the extended set. If, however, the (adjacent) balanced alternative is indeed perceived as the least extreme option (as predicted by hypothesis 1a), it will decrease the likelihood that the middle option will be perceived as the compromise alternative. As a result, the compromise effect in this scenario should be less pronounced than when none of the options is balanced.

The second scenario depicts a case in which the balanced option becomes one of the adjacent options in the extended choice set (i.e., $\left.\left\{x, y_{B}\right\} \rightarrow\left\{z, x, y_{B}\right\}\right)$. Consistent with the predictions made by hypothesis $1 \mathrm{a}$, the adjacent balanced option $y_{B}$ in the extended set is likely to be perceived as the compromise alternative, thus decreasing the relational extremeness aversion that would have led to the selection of the middle option as the compromise alternative. Therefore, it is predicted that, in this scenario, the compromise effect associated with the selection of the middle alternative will be less pronounced than when neither of the options is balanced.

The third scenario examines the question of what happens when the balanced alternative becomes the middle option in the extended choice set. Hypothesis $1 \mathrm{~b}$ argued that attribute balance will increase the likelihood that the middle option will be viewed as the compromise alternative. At the same time, the attribute-balance effect is also likely to exist in the core set as well (hypothesis 2). As a result, the incremental compromise effect associated with the scaleequivalence properties of the middle option is likely to be a function of the relative strength of the attribute-balance effects in the binary and the trinary sets. Therefore, no specific prediction is made with respect to the relative strength of the compromise effect in this scenario.

The above predictions can be expressed more formally as follows:

H3: The compromise effect, associated with the increase in the relative share of the middle option when an adjacent alternative is added to the choice set (i.e., $\{x, y\} \rightarrow\{x, y, z\}$ ), is contingent on the attribute balance of choice alternatives as follows:

H3a: The compromise effect will be greater when no balance alternative is present than when the adjacent alternative added to the core set is balanced (i.e., $\{x, y\} \rightarrow\left\{x, y, z_{B}\right\}$ ).

H3b: The compromise effect will be greater when no balance alternative is present than when one of the options in the core set is balanced and this option becomes adjacent after a third option is added to the set (i.e., $\left\{x, y_{B}\right\} \rightarrow\left\{z, x, y_{B}\right\}$ ).

To summarize, two different views of extremeness aversion were outlined. The relational view of extremeness aversion predicts that in the choice set $\{x, y, z\}$, where the attributes of $y$ are between those of $x$ and $z$ (i.e., $x_{1}<y_{1}<$ $z_{1}$ and $x_{2}>y_{2}>z_{2}$ ), $y$ will be perceived as the least extreme, compromise option. In contrast, the attribute-balance argument advanced in this article predicts that an alternative with identical values on both attributes is likely to be perceived as the least extreme option regardless of its relational properties. These predictions are tested in the experiment described next.

\section{EXPERIMENT 1}

\section{Method}

Three hundred and sixty midwestern university students were randomly assigned to the conditions of a 2 (set size: two vs. three options) $\times 2$ (balanced option: present vs. not present) $\times 2$ (location of the balanced option in the triple set: adjacent vs. middle) $\times 3$ (product category: cordless phone, wine, and sunscreen) mixed design. The size of the choice set and the presence of a balanced option were manipulated between subjects, and the other two factors were manipulated within subjects. The product categories used in this experiment are similar to those used in prior behavioral research (e.g., Lynch and Ariely 2000; Nowlis and Simonson 1996).

The stimuli design was consistent with a paradigm used in prior research (Huber, Payne, and Puto 1982; Ratneshwar, Shocker, and Stewart 1987; Simonson 1989). Respondents were presented with choice sets consisting of either two or three alternatives. Each of the alternatives was described by two attributes with values such that neither option dominated the other(s) on both dimensions. To avoid potential pricequality inferences, options were said to be equally priced.

Alternatives were described on attributes using identical scales. Across product categories, three different attribute metrics were used: numeric ratings on a 100-point scale (cordless phone), stars on a five-star scale (wine), and circles with five different levels of fill (sunscreen). Experimental choice sets were composed of either two or three options as shown in figure 3: $\mathrm{BCD}, \mathrm{CD}, \mathrm{CDE}, \mathrm{DE}, \mathrm{DEF}, \mathrm{B}^{\prime} \mathrm{C}^{\prime} \mathrm{D}^{\prime}$, $\mathrm{C}^{\prime} \mathrm{D}^{\prime}$, and $\mathrm{C}^{\prime} \mathrm{D}^{\prime} \mathrm{E}^{\prime}$. Product categories, attributes, and attribute values used in this experiment are given in appendix A. To test the validity of the experimental hypotheses, different combinations of these sets were compared (see results section for more detail).

Participants were told that the study examined product preferences and were asked to consider three choice sets from different product categories. Choice sets were presented in the following order: cordless phone, wine, and sunscreen. For each of the product categories, respondents were asked to select one of the alternatives. Some of the 
FIGURE 3

RELATIONAL PROPERTIES OF CHOICE ALTERNATIVES USED AS STIMULI

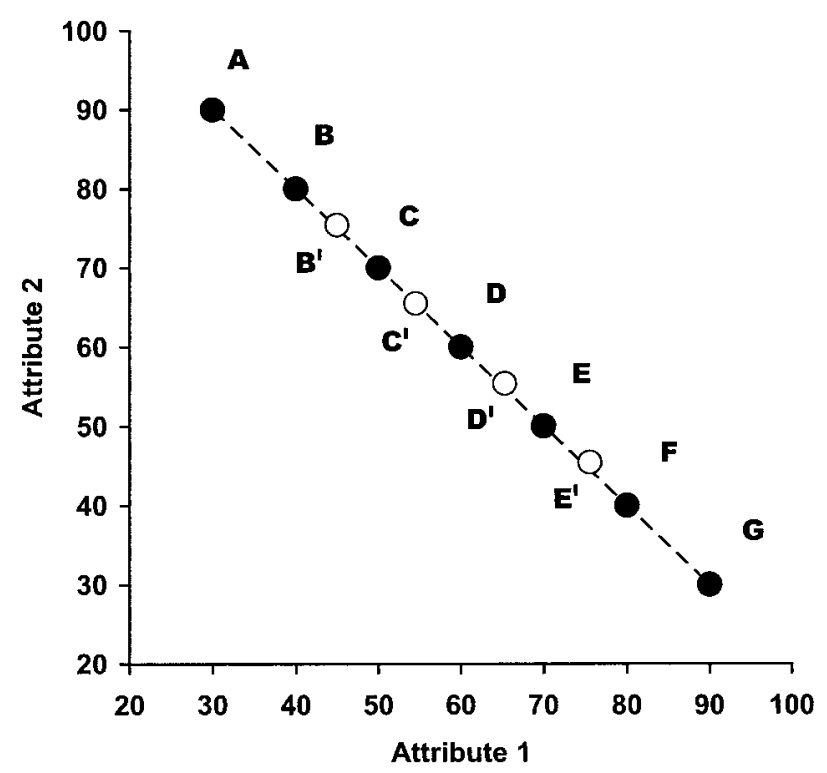

NotE.-More detail on product categories, attributes, and attribute values used in experiments is given in appendix $A$ (experiment 1 ) and appendix $B$ (experiment 2). Options A and $G$ were available in experiment 2 only. Option $D$ is the balanced alternative. Specific attribute values varied across product categories; values shown here are from the toothpaste category used in experiment 2 (app. B).

respondents were also asked to indicate which of the alternatives they perceived to be the easiest to justify. At the end of the experiment respondents were debriefed and paid for participating.

\section{Results}

Attribute-Balance Effects in Trinary Choice Sets. Based on the presence and the relative position of the balanced alternative, there were three types of choice sets: sets without a balanced alternative $\left(\mathrm{B}^{\prime} \mathrm{C}^{\prime} \mathrm{D}^{\prime}\right.$ and $\left.\mathrm{C}^{\prime} \mathrm{D}^{\prime} \mathrm{E}^{\prime}\right)$, sets with an adjacent balanced alternative (BCD and $\mathrm{DEF}$ ), and a set in which the balanced alternative was the middle option (CDE). In this context, hypothesis 1 was tested by analyzing the dispersion of respondents' selections, indicating (1) the most attractive option (the option selected by the respondents) and (2) the option perceived to be easiest to justify.

The dispersion of the choice shares of the options, summarized in table 1 , shows that the balanced alternative had the highest choice share, ranging from $57.1 \%$ to $60.4 \%$ across the experimental conditions. In the presence of an adjacent balanced alternative, the choice share of the middle option was only $21.1 \%$ (BCD and DEF combined; $n=$ $270)$, compared to $44.4 \%\left(\mathrm{~B}^{\prime} \mathrm{C}^{\prime} \mathrm{D}^{\prime}\right.$ and $\mathrm{C}^{\prime} \mathrm{D}^{\prime} \mathrm{E}^{\prime}$ combined; $n=288)$ for choices from sets without a balanced alternative $\left(\chi^{2}(1)=33.07, p<.001\right)$. In contrast, when the mid- dle option was also balanced, its choice share was $58.7 \%$ $(n=126)$, significantly higher than for choices from sets without a balanced alternative $\left(\chi^{2}(1)=7.15, p<.01\right)$. These findings are consistent with hypotheses $1 \mathrm{a}$ and $1 \mathrm{~b}$.

The dispersion of respondents' perceptions of the ease of justifying their choice followed a similar pattern. In particular, in sets with an adjacent balanced alternative, only $23.7 \%$ of the respondents indicated that the middle option was easier to justify, compared to $57.0 \%$ in sets without a balanced alternative $\left(\chi^{2}(1)=23.22, p<.001\right)$. However, when the balanced alternative was also the middle option, it was selected by $88.4 \%$ of the respondents as being the easiest to justify $\left(\chi^{2}(1)=11.71, p<.01\right)$. These findings are also consistent with the predictions made by hypotheses $1 \mathrm{a}$ and $1 \mathrm{~b}$.

Attribute-Balance Effects in Binary Choice Sets. The data show that, in choices from binary sets (CD and DE), the share of the balanced option was $65.1 \%$ of respondents, compared to $54 \%$, the share of the more popular option in the set without a balanced alternative $\left(\chi^{2}(1)=3.13, p<\right.$ $.10)$. The balanced option was also more likely to be perceived as being easier to justify. Thus, $77.3 \%$ of respondents indicated that the balanced option was easier to justify, whereas when neither of the options was balanced, the share of the option identified as being easier to justify was $58.5 \%$ $\left(\chi^{2}(1)=36.08, p<.05\right)$. Considered together, these data support the predictions outlined in hypothesis 2 .

Attribute Balance and the Compromise Effect. The compromise effect is typically measured by comparing the relative shares of choice alternatives between the core and the extended sets. Thus, if $P(y ; x, z)$ is the share of $y$ relative to options $x$ and $z$ in a selection made from the set $\{x, y$, $z\}$, then $P_{z}(y ; x)$ is the share of $y$ relative to $x$ in a selection made from the set $\{x, y, z\}$, where

$$
P_{z}(y ; x)=\frac{P(y ; x, z)}{P(y ; x, z)+P(x ; y, z)} .
$$

\section{TABLE 1}

CHOICE SHARES OF DECISION ALTERNATIVES AS A FUNCTION OF ATTRIBUTE BALANCE (EXPERIMENT 1)

\begin{tabular}{lccccc}
\hline \hline Balanced option & Sets & $N$ & $\begin{array}{c}\mathrm{P}(x ; y, z) \\
(\%)\end{array}$ & $\begin{array}{c}\mathrm{P}(y ; x, z) \\
(\%)\end{array}$ & $\begin{array}{c}\mathrm{P}(z ; x, y) \\
(\%)\end{array}$ \\
\hline Adjacent & $\mathrm{BCD}$ & 144 & 22.9 & 16.7 & $\mathbf{6 0 . 4}$ \\
Middle & $\mathrm{CDE}$ & 126 & 20.6 & $\mathbf{5 8 . 7}$ & 20.6 \\
Adjacent & $\mathrm{DEF}$ & 126 & $\mathbf{5 7 . 1}$ & 26.2 & 16.7 \\
Not present & $\mathrm{B}^{\prime} \mathrm{C}^{\prime} \mathrm{D}^{\prime}$ & 144 & 20.8 & 43.8 & 35.4 \\
Not present & $\mathrm{C}^{\prime} \mathrm{D}^{\prime} \mathrm{E}^{\prime}$ & 144 & 34.0 & 45.1 & 20.8 \\
\hline
\end{tabular}

NOTE.-The choice set composition is consistent with the representation given in fig. 3. The choice shares of the balanced alternative are given in boldface. Notation used is as follows: $P(x ; y, z)$ denotes the share of $x$ relative to options $y$ and $z$ in a selection made from the set $\{x, y, z\}$. In the context of set $\mathrm{ABC}, P(x ; y, z)$ refers to the share of option $\mathrm{A}, P(y ; x, z)$ refers to the share of option $\mathrm{B}$, and $P(z ; x, y)$ refers to the share of option $\mathrm{C}$. The data from binary sets $C D, D E$, and $C^{\prime} D^{\prime}$ are reported in the text. Reported results are averaged across product categories. 
Consistent with prior research, the compromise effect is measured in terms of the changes in the relative share associated with adding an adjacent alternative to the choice set [i.e., $\left.P_{z}(y ; x)-P(y ; x)\right]$. This measure was used to examine whether the compromise effect is moderated by the presence of a balanced alternative, as predicted in hypotheses $3 a$ and $3 b$.

The dispersion of differences in the relative share of the middle option in the binary and the trinary sets is given in table 2. In the absence of a balanced alternative, the data show a positive compromise effect, whereby adding an adjacent alternative to a binary set increases the relative share of the middle option in the extended set $(\Delta P=6.1 \%)$. This finding is consistent with prior research. The presence of an adjacent balanced alternative, however, had a significant impact on the direction and the strength of the compromise effect. In particular, adding an adjacent balanced alternative to a binary set (i.e., $\{x, y\} \rightarrow\left\{x, y, z_{B}\right\}$ ) resulted in a significant decrease in the relative share of the middle option $\left(\Delta P=-12.3 \%\right.$ vs. $\left.\Delta P=6.1 \% ; \chi^{2}(1)=5.73 ; p<.05\right)$. Thus, the relative share of the middle option was actually lower in the extended set than in the core set-a finding consistent with hypothesis $3 \mathrm{a}$.

When an adjacent alternative was added to a set in which one of the options was balanced (i.e., $\left\{x, y_{B}\right\} \rightarrow\left\{z, x, y_{B}\right\}$ ), the relative share of the middle option also decreased by $\Delta P=-8.8 \%$. This decrease in the relative share of the middle option was significant $(\Delta P=-8.8 \%$ vs. $\Delta P=$ $\left.6.1 \% ; \chi^{2}(1)=4.57 ; p<.05\right)$, lending support to hypothesis $3 b$. Finally, when an adjacent option was added to a core set with a balanced alternative that became the middle option in the extended set (i.e., $\left\{x, y_{B}\right\} \rightarrow\left\{x, y_{B}, z\right\}$ ), the compromise effect measured by the increase in the share of the middle alterative was positive $(\Delta P=9.3 \%)$ and not significantly different from the base scenario in which none of the options is balanced $\left(\Delta P=9.3 \%\right.$ vs. $\Delta P=6.1 \% ; \chi^{2}(1)<1$, NS $)$.

\section{Discussion}

The experimental data were consistent with the proposition that the presence of a balanced alternative is likely to have a significant impact on the selection of the compromise option and the compromise effect. In particular, the balanced option in both binary and trinary sets was more often preferred than the other option(s) in the set even when the balanced option was the adjacent option rather than the middle option. Furthermore, the middle-option compromise effect, documented by prior research, was found to be less pronounced in the presence of an adjacent balanced alternative than when the balanced alternative was either not present or was the middle option in the extended set.

The data presented thus far, although consistent with the proposition that the balanced alternative is likely to be perceived as the compromise option, do not offer direct evidence that the observed effects are indeed caused by individuals' extremeness aversion. One strategy to test the link between attribute-balance effects and extremeness aversion is to vary extremeness aversion across respondents and, in this context, examine its impact on the attribute-balance effects. If the observed attribute-balance effects are indeed a function of individuals' extremeness aversion, these effects are likely to be more pronounced for more extremenessaverse individuals. This prediction can be summarized more formally as follows:

H4: The effect of attribute balance on the selection of the compromise option is a function of a consumer's extremeness aversion. In particular, the attribute-balance effect will be greater when an individual's extremeness aversion is high rather than low.

An interesting data pattern observed in the first experiment is that the binary sets without a balanced alternative displayed a pattern similar to the sets with a balanced alternative. In particular, in set $\mathrm{B}^{\prime} \mathrm{C}^{\prime} \mathrm{D}^{\prime}$, the extreme option $\mathrm{D}^{\prime}$, which is closest to the attribute-balance line, fares better than the extreme option $\mathrm{B}^{\prime}\left(35 \%\right.$ vs. $21 \%$ ), and in set $\mathrm{C}^{\prime} \mathrm{D}^{\prime} \mathrm{E}^{\prime}$, the extreme option $\mathrm{C}^{\prime}$ fares better than the extreme option $\mathrm{E}^{\prime}(34 \%$ vs. $21 \%$ ). Similarly, when choosing from sets BC and $\mathrm{EF}, 63.9 \%$ of the respondents selected options $\mathrm{C}$ and $\mathrm{E}$, respectively, each of which was closest to the attributebalance line. This observation raises the question of whether attribute-balance effects can also be observed in sets without a balanced alternative.

The attribute-balance argument proposes that individuals evaluate an option's extremeness with respect to its proximity to the attribute-balance line, such that the balanced alternative is perceived to be the least extreme alternative. If this theory is correct, then it could also be argued that attribute-balance effects would be observed even in the ab-

TABLE 2

THE COMPROMISE EFFECT AS A FUNCTION OF ATTRIBUTE BALANCE (EXPERIMENT 1)

\begin{tabular}{|c|c|c|c|c|c|}
\hline \multirow[b]{2}{*}{ Balanced option } & \multicolumn{2}{|c|}{ Choice set composition } & \multicolumn{2}{|c|}{$P$} & \multirow{2}{*}{$\begin{array}{l}\triangle P \\
(\%)\end{array}$} \\
\hline & Binary set & Trinary set & Binary set (\%) & Trinary set (\%) & \\
\hline Adjacent, trinary set & $\mathrm{BC}, \mathrm{EF}$ & $\mathrm{BCD}, \mathrm{DEF}$ & 63.9 & 51.6 & -12.3 \\
\hline Adjacent, both sets & $C D, D E$ & $\mathrm{BCD}, \mathrm{DEF}$ & 35.3 & 26.5 & -8.8 \\
\hline Middle & CD & CDE & 64.7 & 74.0 & 9.3 \\
\hline Not present & $\mathrm{C}^{\prime} \mathrm{D}^{\prime}$ & $\mathrm{B}^{\prime} \mathrm{C}^{\prime} \mathrm{D}^{\prime}, \mathrm{C}^{\prime} \mathrm{D}^{\prime} \mathrm{E}^{\prime}$ & 50.0 & 56.1 & 6.1 \\
\hline
\end{tabular}

NotE. $-P$ is the relative share of the middle option, and $\Delta P$ is the change in the relative share of the middle option (the compromise effect). 
sence of a balanced alternative, as long as options vary in their proximity to the attribute-balance line. To illustrate, consider the choice set $\mathrm{ABC}$, shown in figure 3 . Because options A $(30,90), B(40,80)$, and C $(70,50)$ vary in their proximity to the attribute-balance line, it could be argued that option $\mathrm{C}$, which is the closest to the attribute-balance line, is likely to be perceived as the least extreme option. This proposition can be summarized as follows:

H5: The attribute-balance effect can occur even in the absence of a balanced alternative, with the option closest to the attribute-balance line acting as a balanced alternative.

These hypotheses are tested in the next experiment.

\section{EXPERIMENT 2}

The goal of this experiment was to provide further support for hypotheses 1-3, as well as to test the propositions outlined in hypotheses 4 and 5 .

\section{Method}

Two hundred and eighty-one midwestern university students were assigned to the conditions of a 2 (set size: two vs. three options) $\times 2$ (balanced option: present vs. not present) $\times 2$ (location of the balanced option in the triple set: adjacent vs. middle) $\times 2$ (extremeness aversion: high vs. low) mixed factorial design. These factors were tested across four product categories (digital camera, printer, toothpaste, and mouthwash).

The stimuli design was similar to that of experiment 1 , in that respondents were presented with choice sets consisting of either two or three alternatives, each described on two attributes. As in the first experiment, options were said to be equally priced to avoid potential price-quality inferences. The specific attribute values of choice alternatives are given in appendix B. All attribute values were given as ratings on a 100-point scale. To account for possible effects associated with a specific set of numeric values (e.g., 50/50 vs. 60/60), the attribute ratings of the choice options were varied across product categories. Thus, the attribute values of the balanced option varied from 50/50 when the stimulus was a digital camera to $65 / 65$ when the stimulus was a printer. Experimental choice sets consisted of either two or three options as follows: $\mathrm{ABC}, \mathrm{BC}, \mathrm{BCD}, \mathrm{CD}, \mathrm{CDE}, \mathrm{DE}$, DEF, EF, EFG, $\mathrm{B}^{\prime} \mathrm{C}^{\prime} \mathrm{D}^{\prime}, \mathrm{C}^{\prime} \mathrm{D}^{\prime}$, and $\mathrm{C}^{\prime} \mathrm{D}^{\prime} \mathrm{E}^{\prime}$. The relational properties of the alternatives are shown in figure 3. Set size (binary vs. trinary) was varied between subjects, and the order in which different choice sets was given to respondents was randomized.

Respondents were told that the study was about product preferences and were given four different choice sets-one per product category. Respondents were then asked to indicate which of the alternatives they would consider to be the safe/compromise option. To manipulate participants' extremeness aversion, a projective technique was used in which participants were asked to predict the choice behavior of a person who either does or does not like to make risky extreme decisions. Similar projective tasks have been successfully used in prior research (Kassarjian 1974; Prelec and Loewenstein 1998; Simonson 1989). Some of the participants were asked to indicate which of the available alternatives was most likely to be chosen by a person who likes to make risky/extreme decisions, while others were asked the same question with respect to a person who does not like to make risky/extreme decisions.

Next, respondents were asked to rate the alternatives on a 10-point scale in terms of how easy it was to justify the choice of each alternative. Finally, they were asked to write down the rationale for their answer to the choice question (predicting the choice of a person who does/does not like to make extreme decisions). Each respondent answered four questions, one per product category.

\section{Results}

Attribute-Balance Effects in Choice. The data summarized in table 3 show that, in the presence of an adjacent balanced alternative (option D in sets BCD and DEF), only $20.1 \%$ of the respondents selected the middle alternative as the compromise choice, whereas $65.5 \%$ selected the adjacent balanced alternative as the compromise option $(n=154)$. The share of responses selecting the middle option as the compromise alternative in this condition was significantly lower than in the absence of a balanced alternative $55.8 \%$ vs. $\left.20.1 \% ; \chi^{2}(1)=44.44 ; p<.001\right)$. Finally, when the middle alternative was balanced (option D in the set CDE), it was selected by $77.5 \%$ of the respondents $(n=79)$ as the compromise option-significantly higher than in cases when none of the alternatives was balanced $(55.8 \%$ vs. $77.5 \%$; $\left.\chi^{2}(1)=8.14 ; p<.005\right)$. These effects were consistent across product categories. The data pattern for ease of justification was similar to that reported in the first experiment. These findings further support hypotheses $1 \mathrm{a}$ and $1 \mathrm{~b}$.

The data from binary sets displayed a pattern similar to the findings reported in the first experiment. In particular, $72.4 \%$ of respondents $(n=156)$ choosing from sets CD and DE selected the balanced option D as the compromise alternative; in contrast, when neither of the options was balanced, the share of the option identified as the compro-

TABLE 3

SHARE OF THE OPTION PERCEIVED TO BE THE COMPROMISE ALTERNATIVE (EXPERIMENT 2)

\begin{tabular}{|c|c|c|c|c|}
\hline Choice sets & $N$ & $\begin{array}{c}P(x ; y, z) \\
(\%)\end{array}$ & $\begin{array}{c}P(y ; x, z) \\
(\%)\end{array}$ & $\begin{array}{c}P(z ; x, y) \\
(\%)\end{array}$ \\
\hline BCD & 77 & 13.0 & 22.1 & 64.9 \\
\hline CDE & 80 & 7.5 & 77.5 & 15.0 \\
\hline DEF & 77 & 66.2 & 18.2 & 15.6 \\
\hline $\mathrm{B}^{\prime} \mathrm{C}^{\prime} \mathrm{D}^{\prime}$ & 112 & 9.8 & 54.5 & 35.7 \\
\hline $\mathrm{C}^{\prime} \mathrm{D}^{\prime} \mathrm{E}^{\prime}$ & 112 & 25.9 & 57.1 & 17.0 \\
\hline
\end{tabular}

choice shares of the balanced alternative are given in boldface. 
TABLE 4

CHOICE SHARES OF DECISION ALTERNATIVES AS A FUNCTION OF ATTRIBUTE BALANCE AND EXTREMENESS AVERSION (EXPERIMENT 2)

\begin{tabular}{|c|c|c|c|c|c|c|c|c|c|}
\hline \multirow[b]{2}{*}{ Balanced alternative } & \multirow[b]{2}{*}{ Sets } & \multicolumn{4}{|c|}{ High extremeness aversion } & \multicolumn{4}{|c|}{ Low extremeness aversion } \\
\hline & & $N$ & $\begin{array}{c}P(x ; y, z) \\
(\%)\end{array}$ & $\begin{array}{c}P(y ; x, z) \\
(\%)\end{array}$ & $\begin{array}{c}P(z ; x, y) \\
(\%)\end{array}$ & $N$ & $\begin{array}{c}P(x ; y, z) \\
(\%)\end{array}$ & $\begin{array}{c}P(y ; x, z) \\
(\%)\end{array}$ & $\begin{array}{c}P(z ; x, y) \\
(\%)\end{array}$ \\
\hline Adjacent & BCD & 43 & 18.6 & 16.3 & 65.1 & 33 & 60.6 & 6.1 & 33.3 \\
\hline Middle & CDE & 45 & 6.7 & 77.8 & 15.6 & 35 & 51.4 & 8.6 & 40.0 \\
\hline Adjacent & $\mathrm{DEF}$ & 43 & 72.1 & 13.9 & 13.9 & 32 & 25.0 & 9.4 & 65.6 \\
\hline Not present & $\mathrm{B}^{\prime} \mathrm{C}^{\prime} \mathrm{D}^{\prime}$ & 60 & 13.3 & 50.0 & 36.7 & 52 & 51.9 & 9.6 & 38.5 \\
\hline Not present & $\mathrm{C}^{\prime} \mathrm{D}^{\prime} \mathrm{E}^{\prime}$ & 60 & 25.0 & 58.3 & 16.7 & 52 & 42.3 & 9.6 & 48.1 \\
\hline
\end{tabular}

NOTE.-The aggregated shares of the middle option in the high-extremeness condition were as follows: $P(y ; x, z)=15.1 \%$ for sets BCD and DEF and $P(y ; x, z)=54.2 \%$ for sets $B^{\prime} C^{\prime} D^{\prime}$ and $C^{\prime} D^{\prime} E^{\prime}$. The choice shares of the balanced alternative are given in boldface.

mise option was $57.9 \%(n=114)$. This difference was significant $\left(\chi^{2}(1)=30.61, p<.001\right)$, lending further support to hypothesis 2 .

Compromise as a Reason for Choice. Respondents' self-reported choice reasons (e.g., Ericsson and Simon 1980) offer further insight into the decision processes leading to the selection of the compromise alternative. In particular, respondents' choice reasons were classified into three categories: (1) primary attribute, (2) compromise, and (3) other. The primary-attribute category included responses such as "picture clarity is more important" and "quality over speed." The compromise category included responses such as "it is the middle option," "get both benefits equally," and "balance in ratings." Responses that could not be classified in either category were listed in the "other" category. Overall, $84 \%$ of all responses offered a rationale that was classified into one of the two focal categories; the remaining $16 \%$ either did not provide any rationale for their decisions or could not be uniquely classified.

Qualitative analysis of the compromise responses shows that a number of respondents explicitly identified attribute balance as a reason for their choice. To illustrate, attributebalance reasons included: "it is clearly an excellent choice due to being at parity and parity = comfort," "because it's 50-50, you can't go wrong," "equally represented," "equal ratings for both should prevent risk," "betweenness," "it's the compromise choice," "covers both bases," "the happy medium," "ratings are identical, no sacrifices made." Quantitative analysis of these reasons $(n=379)$ shows that respondents were significantly more likely to use the compromise rationale when a balanced alternative was present than when it was not present (sets BCD, CDE, and DEF vs. $\mathrm{B}^{\prime} \mathrm{C}^{\prime} \mathrm{D}^{\prime}$ and $\left.\mathrm{C}^{\prime} \mathrm{D}^{\prime} \mathrm{E}^{\prime} ; \chi^{2}(1)=10.67 ; p<.005\right)$.

Finally, the analysis of respondents' reasoning for choices made from sets with a balanced alternative (BCD, CDE, and DEF) shows that respondents who selected an adjacent balanced alternative (option D in the sets BCD and DEF) were more likely to use compromise as the rationale to justify their choices than were respondents who selected an adjacent nonbalanced option (option B in the set BCD and option F in the set DEF; $76.6 \%$ vs. $\left.20 \%, \chi^{2}(1)=15.34 ; p<.001\right)$.
Similarly, respondents who selected the balanced middle option (option D in the set CDE) were more likely to use compromise as the rationale to justify their choices than respondents who selected the nonbalanced middle option (option $\mathrm{C}$ in the set $\mathrm{BCD}$ and option $\mathrm{E}$ in the set DEF; $95.8 \%$ vs. $\left.20 \%, \chi^{2}(1)=15.08 ; p<.001\right)$. Overall, these data further support hypotheses $1 \mathrm{a}$ and $1 \mathrm{~b}$ by showing that respondents were likely to perceive the balanced option as the compromise alternative.

Attribute Balance and Extremeness Aversion. A further test of the attribute-balance hypothesis is given by analyzing respondents' predictions of choices by a person with either a high or a low level of extremeness aversion. The data given in table 4 show a distinct pattern of dispersion of choice shares across the two extremeness-aversion conditions.

In particular, respondents in the high extremeness-aversion condition were most likely to choose the balanced option $\mathrm{D}$ regardless of its relative position in the set $(M=$ $65.1 \%, M=77.8 \%$, and $M=72.1 \%$, respectively). The middle option was most preferred only when it was also balanced, but not when an adjacent balanced alternative was also present in the set $\left(77.8 \%\right.$ vs. $15.1 \% ; \chi^{2}(1)=40.47$; $p<.001)$. Comparing the choice share of the middle alternative in sets with and without a balanced alternative further shows that the middle option was significantly less likely to be chosen in the presence of an adjacent balanced alternative $\left(54.2 \%\right.$ vs. $\left.15.1 \% ; \chi^{2}(1)=28.85 ; p<.001\right)$ and significantly more likely to be chosen when it was balanced $\left(54.2 \%\right.$ vs. $\left.77.8 \% ; \chi^{2}(1)=7.27 ; p<.01\right)$. These data are consistent with the predictions made by hypotheses $1 \mathrm{a}$ and $1 b$.

Comparing the choice share of the middle alternative in sets with and without a balanced option further shows that the attribute-balance effect was more pronounced in the high than in the low extremeness-aversion condition $\left(\chi^{2}(1)=\right.$ $6.02 ; p=.01)$. For choices from binary sets, the preference for the balanced option was a function of extremeness aversion as well. In particular, the balanced option was chosen by $74.3 \%$ of respondents $(n=78)$ in the high extremenessaversion condition and by only $28.2 \%$ of the respon- 
dents $(n=78)$ in the low extremeness-aversion condition $\left(\chi^{2}(1)=30.61 ; p<.001\right)$. These findings are consistent with the predictions made by hypothesis 4 .

Attribute Balance and the Compromise Effect. The dispersion of differences in the relative shares of the middle option in the binary and the trinary sets from the high extremeness-aversion condition was similar to the data reported in the first experiment. The data summarized in table 5 show that, in the absence of a balanced alternative, the share of the middle alternative was higher in the context of the trinary than in the binary set $(\Delta P=13.8 \%)$ - a finding consistent with prior research.

When an adjacent balanced alternative was present only in the trinary set (i.e., $\{x, y\} \rightarrow\left\{x, y, z_{B}\right\}$ ), however, the compromise effect was negative: the share of the middle option was lower in the trinary set than in the binary set $(\Delta P=$ $-20.9 \%$ ). This difference in the shares of the middle option was also significantly different from the case where none of the options was balanced $(\Delta P=-20.9 \%$ vs. $\Delta P=$ $\left.13.8 \% ; \chi^{2}(1)=7.44 ; p<.01\right)$. These data are consistent with hypothesis $3 \mathrm{a}$. The compromise effect was also negative when an adjacent balanced alternative was present in both the binary and the trinary sets (i.e., $\left\{x, y_{B}\right\} \rightarrow$ $\left.\left\{z, x, y_{B}\right\}\right)$, whereby the share of the middle option was again lower in the trinary than in the binary set $(\Delta P=-7.5 \%)$. In this case, the compromise effect was significantly different from the base scenario in which none of the options was balanced $\left(\Delta P=-7.5 \%\right.$ vs. $\Delta P=13.8 \% ; \chi^{2}(1)=$ $4.37 ; p<.05)$, a finding consistent with hypothesis $3 \mathrm{~b}$.

A further test of the attribute-balance effect is given by examining respondents' selection of the compromise alternative across binary and trinary sets and then using these data to infer respondents' choices. Because the data pertaining to the selection of the compromise alternative are aggregated across the two extremeness-aversion conditions, using these data to infer choice allows controlling for po- tential biases associated with the extremeness-aversion manipulation. The pattern of the compromise data shown in table 5, panel B, is consistent with the choice data reported earlier. The statistics measuring the strength of the compromise effect across different conditions were consistent with the statistics reported earlier in this section, lending further support to hypotheses $3 \mathrm{a}$ and $3 \mathrm{~b}$.

Attribute-Balance Effects without a Balanced Alternative. An important question raised by hypothesis 5 is whether attribute-balance effects can occur in the absence of a balanced alternative, with an option's proximity to the attribute-balance line serving as a proxy for balance. In this context, the data show that respondents were more likely to view options closer to the attribute-balance line as the compromise alternative. Thus, in sets $\mathrm{ABC}$ and $\mathrm{EFG}$, the share of the option closest to the attribute-balance line was $51.0 \%$ $(n=149)$, whereas the share of the option farthest away from the attribute-balance line was only $13.4 \%$. In contrast, in sets $\mathrm{B}^{\prime} \mathrm{C}^{\prime} \mathrm{D}^{\prime}$ and $\mathrm{C}^{\prime} \mathrm{D}^{\prime} \mathrm{E}^{\prime}$ the difference was less pronounced (13.4\% vs. $30.8 \% ; n=224)$. Furthermore, in sets $\mathrm{B}^{\prime} \mathrm{C}^{\prime} \mathrm{D}^{\prime}$ and $\mathrm{C}^{\prime} \mathrm{D}^{\prime} \mathrm{E}^{\prime}$, the middle option was identified as the compromise alternative by $55.8 \%$ of respondents $(n=224)$, compared to only $35.6 \%$ of respondents $(n=149)$ in sets $\mathrm{ABC}$ and $\mathrm{EFG}\left(\chi^{2}(1)=8.66 ; p<.005\right)$. The data from the binary sets BC and EF reveal a similar pattern: $69 \%$ $(n=142)$ of the respondents identified the option closest to the attribute-balance line as the compromise alternative, compared to $57.9 \%(n=114)$ of respondents choosing from set $\mathrm{C}^{\prime} \mathrm{D}^{\prime}\left(\chi^{2}(1)=3.38, p<.10\right)$.

The reason-based analysis further shows that a number of individuals used the attribute-balance rationale with respect to the option closest to the attribute-balance line; the reasons given included "lower tradeoff: ratings are closer," "all criteria are closer to average," and "because ratings are more consistent." In particular, $36.2 \%$ of respondents $(n=69)$ who chose options $\mathrm{C}$ or E from sets ABC and

TABLE 5

THE COMPROMISE EFFECT AS A FUNCTION OF ATTRIBUTE BALANCE (EXPERIMENT 2)

\begin{tabular}{|c|c|c|c|c|c|}
\hline \multirow[b]{2}{*}{ Balanced option } & \multicolumn{2}{|c|}{ Choice set composition } & \multicolumn{2}{|c|}{$P$} & \multirow{2}{*}{$\begin{array}{l}\triangle P \\
(\%)\end{array}$} \\
\hline & Binary set & Trinary set & Binary set (\%) & Trinary set (\%) & \\
\hline \multicolumn{6}{|c|}{$\begin{array}{l}\text { A. Compromise effect for respondents in the high } \\
\text { extremeness-aversion condition: }\end{array}$} \\
\hline Adjacent, trinary set & $\mathrm{BC}, \mathrm{EF}$ & $\mathrm{BCD}, \mathrm{DE} \mathrm{F}$ & 69.2 & 48.3 & -20.9 \\
\hline Adjacent, both sets & $\mathrm{CD}, \mathrm{DE}$ & $\mathrm{BCD}, \mathrm{DEF}$ & 25.6 & 18.1 & -7.5 \\
\hline Middle & CD & $\mathrm{CDE}$ & 74.4 & 87.7 & 13.4 \\
\hline Not present & $\mathrm{C}^{\prime} \mathrm{D}^{\prime}$ & $\mathrm{B}^{\prime} \mathrm{C}^{\prime} \mathrm{D}^{\prime}, \mathrm{C}^{\prime} \mathrm{D}^{\prime} \mathrm{E}^{\prime}$ & 50.0 & 63.8 & 13.8 \\
\hline \multicolumn{6}{|c|}{$\begin{array}{l}\text { B. Compromise effect inferred from respondents' } \\
\text { selection of the compromise option: }\end{array}$} \\
\hline Adjacent, trinary set & $B C, E F$ & $\mathrm{BCD}, \mathrm{DEF}$ & 69.0 & 58.4 & -10.6 \\
\hline Adjacent, both sets & $\mathrm{CD}, \mathrm{DE}$ & $\mathrm{BCD}, \mathrm{DEF}$ & 27.6 & 23.5 & -4.1 \\
\hline Middle & CD & CDE & 72.4 & 87.5 & 15.0 \\
\hline Not present & $\mathrm{C}^{\prime} \mathrm{D}^{\prime}$ & $B^{\prime} C^{\prime} D^{\prime}, C^{\prime} D^{\prime} E^{\prime}$ & 50.0 & 64.6 & 14.6 \\
\hline
\end{tabular}

NoTE. $-P$ is the relative share of the middle option, and $\Delta P$ is the difference in the relative shares of the middle option between the binary and trinary sets (the compromise effect). 
EFG used attribute balance as a rationale to support their selection. Similar results were obtained for choices from binary sets BC and EF, where attribute balance was used by $29.9 \%$ of respondents $(n=67)$ as a rationale in support of choosing options $\mathrm{C}$ and $\mathrm{E}$. These data further support the prediction made by hypothesis 5 that the attribute-balance effect can exist even in the absence of a balanced alternative, in which case extremeness aversion is a function of the proximity of an option's attribute ratings to the attributebalance line (hypothesis 5).

\section{Discussion}

The data reported in experiment 2 show that, in the presence of an adjacent balanced alternative, the middle option is less likely to be perceived as the compromise option-an effect consistent with hypotheses 1 and 2 . The data also support hypothesis 3 by demonstrating that the compromise effect is contingent upon the options' balance. More important, the attribute-balance effect was shown to be a function of individuals' extremeness aversion in that the attribute-balance effects were more pronounced for more extremeness-averse individuals. This finding, combined with the analysis of respondents' reasons for choice, lends support to the proposition that attribute-balance effects are a function of extremeness aversion (hypothesis 4). Finally, the data show that attribute-balance effects can occur even in the absence of a balanced alternative, such that the option closest to the attribute-balance line can lead to attributebalance effects as well (hypothesis 5).

An interesting observation made while analyzing respondents' reasons for choice is that a number of respondents referred to the balanced alternative as the "middle" option, even in cases where it was adjacent in relative terms. These data raise the issue of whether consumers' mental representations of choice alternatives are always adequately reflected by the representation given in figure 1 . Thus, it is possible that instead of using an orthogonal representation of choice alternatives, individuals simply align their attribute values. To illustrate, consider the set BCD shown in figure 2. The orthogonal representation positions option $C$ as the middle alternative and options $\mathrm{B}$ and $\mathrm{D}$ as the extremes. When both attributes use identical scales, individuals might simply collapse their scales, anchoring on the relational properties of the choice alternatives. Such representation would also position option $\mathrm{C}$ as the middle alternative and options B and D as the extremes, as shown in figure 4, panel A.

Note, however, that the middle position of option $\mathrm{C}$ in the above example is contingent on individuals aligning attribute scales by matching the relative position of the options on each attribute. An alternative, and perhaps more intuitive approach, is to align attribute scales by using the scale metric as a reference point. Thus, when individuals align attribute scales by matching their scale values instead of anchoring on the relational properties of the choice alternatives, then the balanced option B will appear as the least extreme alternative (fig. 4, panel B). Because these different scale alignments lead to different predictions about which alternative will be perceived as the middle option, manipulating the reference point used to align attribute values of the choice alternatives should also result in variations in the strength of the attribute-balance effect.

In this context, it is proposed that consumers will be more likely to use the relational alignment (fig. 4, panel A) when they compare an option's values across alternatives, one attribute at a time, than when they compare an option's performance across attributes, one alternative at a time. In contrast, consumers will be more likely to use the scalebased alignment of choice alternatives (fig. 4, panel B) when they process the available information by alternative rather than by attribute. Thus, one strategy to test the alignability account for the attribute-balance effect is to manipulate the pattern of information processing: by attribute versus by alternative.

The above discussion implies that focusing individuals' attention on the relational properties of choice alternatives should weaken the attribute-balance effects. In particular, the attribute-balance effect is likely to be more pronounced when the available attribute information is evaluated in a fashion that facilitates within-alternative comparisons of attribute values (e.g., $x_{1}$ vs. $x_{2}, y_{1}$ vs. $y_{2}$, and $z_{1}$ vs. $z_{2}$ ); in contrast, it is likely to be less pronounced when the available attribute information is evaluated in a fashion that facilitates within-attribute comparisons of the options (e.g., $x_{1}$ vs. $y_{1}$ vs. $z_{1}$ and $x_{2}$ vs. $y_{2}$ vs. $z_{2}$ ). This proposition can be summarized more formally as follows:

H6: The attribute-balance effect is contingent on the nature of the decision task. In particular, the attribute-balance effect will be greater when the available information is processed by alternative than when it is processed by attribute.

This prediction is tested in the next experiment.

\section{EXPERIMENT 3}

The goal of this experiment was to test the proposition that the attribute-balance effect is contingent on the nature of the decision task. This was accomplished by introducing a priming task aimed at stimulating attribute-based processing of the available information. The experimental procedure, stimuli, and the results are described in detail in the following sections.

\section{Method}

One hundred and thirty-three midwestern university students were randomly assigned to either the control or the attribute-focus condition. The control condition was identical to the high extremeness-aversion condition from the second experiment. The attribute-focus condition was identical to the control condition except that it was preceded by a priming task.

The priming task presented respondents with a trinary choice set from a different product category (cordless 
FIGURE 4

SCALE ALIGNMENT AND ATTRIBUTE BALANCE IN CHOICE

A. Relational alignment of choice alternatives

Attribute 1

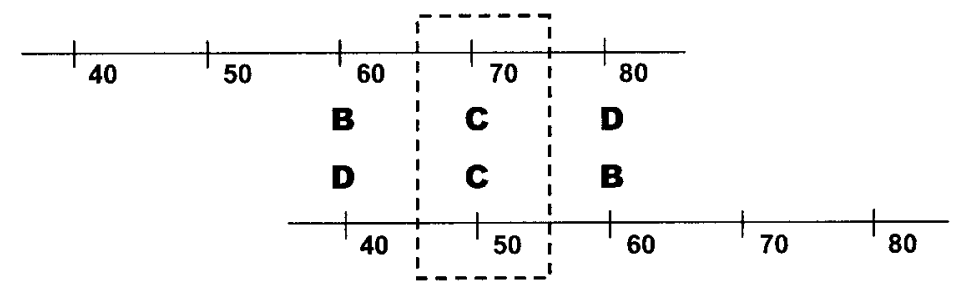

Attribute 2

B. Scale-based alignment of choice alternatives

Attribute 1

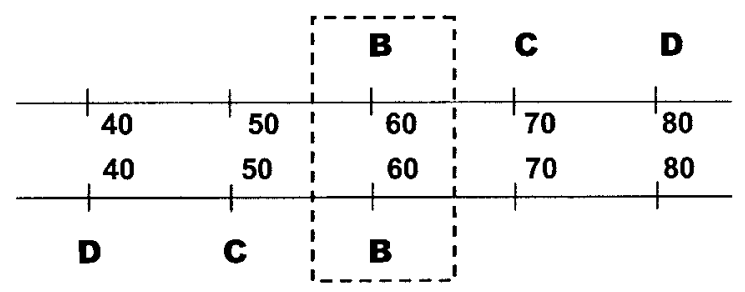

NOTE.-The figure illustrates different linear representations of options B $(60,60), C(70,50)$, and D $(80,40)$ depicted in fig. 2 . Options in panel A are aligned based on their relational properties; in panel $B$, options are aligned by matching the attribute scales. Collapsing the two scales in the first scenario positions $C$ as the middle option. In contrast, collapsing the two attribute scales in the second scenario positions B as the middle option.

phone). The two attributes describing choice alternatives were battery life and sound quality, with ratings as follows: $(70,50),(80,40)$, and $(90,30)$. Respondents were asked to indicate which of the options would be selected (1) by a person who cares exclusively about battery life, (2) by a person who cares exclusively about sound quality, and (3) by a person who cares equally about battery life and sound quality. The goal of this manipulation was to prime respondents to evaluate the available information one attribute at a time and to explicitly compare options' values within each of the attributes. It was expected that this pattern of information evaluation would carry over to the main decision task and increase the likelihood of attribute-based comparisons of the choice alternatives.

The product categories, attributes, and attribute values describing choice alternatives in the main decision task, which followed the attribute-priming manipulation, were the same as those in sets BCD, CDE, and DEF in experiment 2. The rest of the experimental procedure and the dependent variables were the same as in the second study: respondents evaluated four different choice sets and were asked to (1) select the least extreme alternative and (2) rate the alternatives in terms of how easy it was to justify the choice of each option.

\section{Results}

To examine whether and how the choice task moderates the attribute-balance effect, two factors were analyzed: respondents' selection of the compromise option and the perceived ease of justification. The data summarized in table 6 show that, in both conditions, the presence of a balanced alternative had an impact on respondents' selection of the option identified as the compromise alternative. The dispersion of the shares of the middle option in the control condition was consistent with the findings from experiment 2 . The middle option was more likely to be identified as the compromise alternative in cases when it was also balanced than in cases when one of the adjacent options was balanced $\left(68.6 \%\right.$ vs. $19.1 \%, \chi^{2}(1)=33.08$; $p<.001)$. The data from the attribute-focus condition also displayed a significant, although less pronounced, attributebalance effect; thus, $70.8 \%$ of respondents selected the balanced middle alternative as the compromise alternative compared to $46.3 \%$ who selected the middle option when it was not balanced $\left(\chi^{2}(1)=7.48 ; p<.01\right)$. Thus, the attribute-balance effect was observed in both experimental conditions.

Analysis of the relative strength of the attribute-balance effect across the two experimental conditions shows that 
TABLE 6

CHOICE SHARE OF THE COMPROMISE ALTERNATIVE AS A FUNCTION OF ATTRIBUTE BALANCE AND THE DECISION TASK (EXPERIMENT 3)

\begin{tabular}{|c|c|c|c|c|c|c|c|c|c|}
\hline \multirow[b]{2}{*}{ Balanced option } & \multirow[b]{2}{*}{ Sets } & \multicolumn{4}{|c|}{ Control condition } & \multicolumn{4}{|c|}{ Attribute-focus condition } \\
\hline & & $N$ & $\begin{array}{c}P(x ; y, z) \\
(\%)\end{array}$ & $\begin{array}{c}P(y ; x, z) \\
(\%)\end{array}$ & $\begin{array}{c}P(z ; x, y) \\
(\%)\end{array}$ & $N$ & $\begin{array}{c}P(x ; y, z) \\
(\%)\end{array}$ & $\begin{array}{c}P(y ; x, z) \\
(\%)\end{array}$ & $\begin{array}{c}P(z ; x, y) \\
(\%)\end{array}$ \\
\hline Adjacent & BCD & 55 & 9.1 & 18.2 & 72.7 & 48 & .0 & 43.8 & 56.3 \\
\hline Middle & CDE & 51 & 17.6 & 68.6 & 13.7 & 48 & 6.3 & 70.8 & 22.9 \\
\hline Adjacent & DEF & 55 & 69.1 & 20.0 & 10.9 & 43 & 44.2 & 48.8 & 7.0 \\
\hline
\end{tabular}

NOTE.-Reported results are averaged across product categories. The choice shares of the balnced alternative are given in boldface.

this effect was moderated by respondents' processing strategy, as predicted by hypothesis 6 . Thus, respondents given the attribute-priming task were significantly more likely to identify the middle option as the compromise in the presence of an adjacent balanced alternative (relative to the condition when the middle option was balanced) than respondents in the control condition $\left(\chi^{2}(1)=4.76 ; p<.05\right)$.

Further evidence for the moderating effect of the decision task is offered by the data on the ease of justification. Consistent with the data reported in the first two experiments, respondents in the control condition found it easier to justify the selection of the balanced alternative than the selection of any other option in the set $(M=6.96, \mathrm{SD}=2.75 \mathrm{vs}$. $M=5.83, \mathrm{SD}=1.93 ; t(145)=4.79, p<.001)$. In contrast, respondents in the attribute-focus condition did not display significant differences in justifying the selection of the balanced and nonbalanced alternative $(M=6.59$, $\mathrm{SD}=2.65$ vs. $M=6.42, \mathrm{SD}=2.39 ; t(127)<1)$. Comparing the data across the two experimental conditions shows that the difference in perceived ease of justifying the choice of the balanced alternative versus the other options in the set was significant $(\Delta M=1.13$ vs. $\Delta M=.16$; $F(1,272)=9.92, p<.005)$. These findings were consistent with the predictions made by hypothesis 6 .

\section{Discussion}

The data reported in this experiment support the predictions made by hypothesis 6 that the attribute-balance effect is contingent on the nature of the decision task and is more pronounced in the context of a decision task that promotes alternative-based, rather than attribute-based, processing. The data are consistent with the notion that focusing individuals' attention on the relational properties of choice alternatives weakens the attribute-balance effect.

The data reported in experiment 3 also show that, when respondents were asked to focus on the relational properties of the choice alternatives, they were less likely to perceive the balanced alternative as being easier to justify. One possible explanation for this finding is that focusing on the relational properties of the choice alternatives makes the parity of the attribute values of the balanced option less salient, and, as a result, the balanced option does not stand out in any respect; this in turn decreases the likelihood that this option would be used as a reason for choice. This argument is consistent with the notion that, because the middle option is not best on either of the attributes, it does not stand out and, consequently, its choice is more difficult to justify (Simonson 1989; see also Tversky, Sattath, and Slovic 1988).

\section{GENERAL DISCUSSION}

Prior research has shown that the choice probability of a given alternative tends to increase when it becomes the middle option in the set. This finding is based on the assumption that, when an external reference point is not readily available, consumers evaluate the extremeness of the options relative to one another in such a way that the middle option becomes the compromise choice. The research presented in this article builds on these findings and posits that an option can be defined as the compromise alternative based not only on its relational properties but also on the option-specific dispersion of its attribute values. The attribute-balance hypothesis advanced in this article argues that, when choice options are described on attributes using the same metric, the option with equal ratings on both attributes is likely to be perceived as the compromise option even when it is not the middle option in the set.

Data from three studies document the impact of attribute balance on extremeness aversion in choice. It is shown that the attribute balance of an alternative increases both the likelihood of it being perceived as the compromise option as well as its choice share. Both qualitative and quantitative data support the proposition that the selection of the compromise option is affected significantly by the attribute balance of options' in both binary and trinary sets. The data also offer insights into the boundary conditions of the attribute-balance effect, such as individuals' informationprocessing strategy. Overall, these findings suggest that evaluating the extremity of an option's values on a given attribute is a function not only of its relational properties with respect to the other options in the set but also of the dispersion of values of the attributes describing this option.

The proposed account of the attribute-balance effect is 
based on the notion that, when the attributes describing choice alternatives use the same metric, consumers can readily compare an option's performance on different attributes; as a result, an option's extremeness can be evaluated based not only on its relative position in the choice but also on the proximity of its own attribute values. Thus, when the extremeness of a balanced alternative is evaluated based on the proximity of its own attribute values, this option is likely to be selected as the least extreme, compromise alternative even when it is not the middle option in the set. One possible mechanism for such option-specific cross-attribute comparisons, illustrated in figure 4, suggests that individuals might collapse scale-equivalent attributes by matching their end points, which will naturally position the balanced alternative as the compromise option. Since, however, this is only one possible representation of the attribute-balance effect; further research is needed to test alternative accounts as well.

\section{Attribute Balance as a Context Effect}

The relational and the attribute-balance hypotheses share a number of similarities. Both theories run counter to the predictions of the principle of independence of irrelevant alternatives (Luce 1959) and the substitution effect (Huber and Puto 1983). Both theories converge in identifying preference uncertainty as the rationale for a decision maker's selection of the compromise alternative. Furthermore, the effects predicted by both hypotheses are driven by the dispersion of the attribute values describing alternatives in the choice set, rather than by consumers' prior experience with the product category. The key difference concerns the decision processes that lead to the identification of the compromise option. In particular, the attribute-balance effect is distinctive in that, unlike the other context effects, it is independent of the choice context defined by the relational properties of the alternatives. Indeed, the balance of a given option is defined in terms of this option's internal properties, such as the relationship between its own attribute ratings. As a result, attribute balance is likely to produce context effects such as extremeness aversion without being context dependent itself (Chernev, forthcoming). Studying such context-independent effects is a promising area for further research.

\section{Representation Invariance in Choice}

The data reported in this research offer a new perspective on the issue of how consumers represent choice alternatives. Indeed, the commonly used representation of extremeness aversion and the compromise effect (e.g., fig. 1 ) is based on the assumption that consumers' perception of choice alternatives and the relationships between these alternatives can be adequately represented in a multidimensional space, such that metric distances reflect rela- tionships between the alternatives (Shepard 1962; Tversky 1977). The two-dimensional orthogonal representation in particular has been used for illustrating numerous decision phenomena, such as asymmetric dominance (Huber et al. 1982), compromise effect (Simonson 1989), pioneering advantage (Carpenter and Nakamoto 1989), and tradeoff contrast and extremeness aversion (Simonson and Tversky 1992). An implicit assumption in this approach is representation invariance, that is, the assumption that alternative representations of the problem should yield the same result. Research presented in this article questions this assumption by identifying a context in which different representations are likely to lead to different extremeness-aversion predictions, as illustrated in figure 4 . Investigating the nature of consumer representations of choice alternatives and the resulting implications for choice is a promising direction for future research.

\section{Boundary Conditions}

The attribute-balance effect is subject to several boundary conditions; some of these have been identified in this article, while others offer directions for further analysis. Thus, it is important to note that the attribute-balance effect is based on the assumption that consumers do not have readily established attribute preferences and/or that they perceive attributes describing choice alternatives to be of similar importance. Indeed, in cases where consumers have readily established attribute preferences such that one of the attributes is perceived to be of primary importance, the impact of attribute balance is likely to be less pronounced. It is also important to note that the experimental sets were made up of nondominating options so that consumers had to trade off different attributes and/or attribute values. It is likely that, when choices are made from sets with a dominant alternative, the attribute-balance effect will be less pronounced.

Another boundary condition identified in experiment 3 is the dependence of the attribute-balance effect on the information-processing strategy, such that the effect was less pronounced when the relational properties of the choice alternatives were more salient (e.g., when processing the available information by attribute rather than by alternative). Furthermore, for the attribute-balance effect to occur, it is crucial that both attribute scales are readily comparable in terms of their scale range as well as the nature of the scale itself. Thus, attribute-balance effects are less likely to be observed in cases where the scales use different ranges (e.g., 100 -point vs. 7-point rating scales) and/or when the scales have different metrics (e.g., megabytes vs. inches). These boundary conditions are consistent with the proposition, illustrated in figure 4, that individuals need to be able to collapse the attribute scales to yield a representation in which the balanced alternative is perceived as the least extreme option. Further investigation of the boundary conditions is likely to foster the understanding of attribute-balance effects and is a promising direction for further research. 


\section{APPENDIX A}

TABLE A1

ATTRIBUTES AND ATTRIBUTE RATINGS OF PRODUCTS USED IN EXPERIMENT 1

\begin{tabular}{|c|c|c|c|c|c|c|c|c|c|}
\hline \multirow[b]{2}{*}{ Product/attribute } & \multicolumn{9}{|c|}{ Choice alternative } \\
\hline & $B$ & $\mathrm{C}$ & $\mathrm{D}$ & $E$ & $\mathrm{~F}$ & $\mathrm{~B}^{\prime}$ & $\mathrm{C}^{\prime}$ & $D^{\prime}$ & $E^{\prime}$ \\
\hline \multicolumn{10}{|l|}{ Cordless phone: } \\
\hline Battery life & 30 & 40 & 50 & 60 & 70 & 40 & 50 & 60 & 70 \\
\hline Sound quality & 70 & 60 & 50 & 40 & 30 & 70 & 60 & 50 & 40 \\
\hline \multicolumn{10}{|l|}{ Wine: ${ }^{a}$} \\
\hline $\begin{array}{l}\text { Body } \\
\text { Complexity }\end{array}$ & 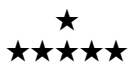 & $\begin{array}{c}\star \star \star \\
\star \star \star \star \star\end{array}$ & $\begin{array}{l}\star \star \star \\
\star \star \star\end{array}$ & $\begin{array}{c}\star \star \star \star \star \\
\star \star\end{array}$ & 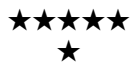 & 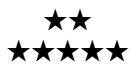 & $\begin{array}{l}\star \star \star \star \\
\star \star \star \star \star\end{array}$ & $\begin{array}{c}\star \star \star \star \star \\
\star \star \star\end{array}$ & $\begin{array}{c}\star \star \star \star \star \star \star ~ \\
\star \star \star\end{array}$ \\
\hline \multirow{2}{*}{\multicolumn{10}{|c|}{$\begin{array}{l}\text { Sunscreen: } \\
\text { UVA protection }\end{array}$}} \\
\hline & & & & & & & & & \\
\hline UVB protection & & & & & & & & & \\
\hline
\end{tabular}

a"Body" is the perception of texture or weight of a wine in the mouth; "complexity" is the perception of multiple layers and nuances of bouquet and flavor. bUVA and UVB are two radiation wavelengths produced by the sun which may damage the skin.

\section{APPENDIX B}

TABLE B1

ATTRIBUTES AND ATTRIBUTE RATINGS OF PRODUCTS USED IN EXPERIMENT 2

\begin{tabular}{|c|c|c|c|c|c|c|c|c|c|c|c|}
\hline \multirow{2}{*}{$\begin{array}{l}\text { Product category/ } \\
\text { attribute }\end{array}$} & \multicolumn{11}{|c|}{ Choice alternative } \\
\hline & A & B & C & $\mathrm{D}$ & $E$ & $\mathrm{~F}$ & $\mathrm{G}$ & $\mathrm{B}^{\prime}$ & $C^{\prime}$ & $D^{\prime}$ & $E^{\prime}$ \\
\hline \multicolumn{12}{|l|}{ Camera: } \\
\hline Picture clarity & 20 & 30 & 40 & 50 & 60 & 70 & 80 & 40 & 50 & 60 & 70 \\
\hline Reliability & 80 & 70 & 60 & 50 & 40 & 30 & 20 & 70 & 60 & 50 & 40 \\
\hline \multicolumn{12}{|l|}{ Printer: } \\
\hline Print quality & 35 & 45 & 55 & 65 & 75 & 85 & 95 & 40 & 50 & 60 & 70 \\
\hline Printing speed & 95 & 85 & 75 & 65 & 55 & 45 & 35 & 70 & 60 & 50 & 40 \\
\hline \multicolumn{12}{|l|}{ Toothpaste: } \\
\hline Breath-freshening & & & & & & & & & & & \\
\hline effectiveness & 30 & 40 & 50 & 60 & 70 & 80 & 90 & 50 & 60 & 70 & 80 \\
\hline Tooth-whitening & & & & & & & & & & & \\
\hline effectiveness & 90 & 80 & 70 & 60 & 50 & 40 & 30 & 80 & 70 & 60 & 50 \\
\hline \multicolumn{12}{|l|}{ Mouthwash: } \\
\hline \multirow{3}{*}{$\begin{array}{l}\text { Germ-killing } \\
\text { effectiveness } \\
\text { Decay-preventing }\end{array}$} & & & & & & & & & & & \\
\hline & 25 & 35 & 45 & 55 & 65 & 75 & 85 & 45 & 55 & 65 & 75 \\
\hline & & & & & & & & & & & \\
\hline effectiveness & 85 & 75 & 65 & 55 & 45 & 35 & 25 & 75 & 65 & 55 & 45 \\
\hline
\end{tabular}

[Dawn Iacobucci served as editor and Stephen Nowlis served as associate editor for this article.]

\section{REFERENCES}

Baylis, Gordon C. and Jon Driver (1992), "Visual Parsing and Response Competition: The Effect of Grouping Factors," Perception and Psychophysics, 51 (2), 145-62.

Carpenter, Gregory S. and Kent Nakamoto (1989), "Consumer Preference Formation and Pioneering Advantage," Journal of Marketing Research, 26 (August), 285-98.

Chernev, Alexander (forthcoming), "Context Effects without a
Context: Attribute Balance as a Reason for Choice," Journal of Consumer Research.

Dhar, Ravi, Stephen M. Nowlis, and Steven J. Sherman (2000), "Trying Hard or Hardly Trying: An Analysis of Context Effects in Choice," Journal of Consumer Psychology, 9 (4), 189-200.

Ericsson, Karl A. and Herbert A. Simon (1980), "Verbal Reports as Data," Psychological Review, 87 (July), 215-51.

Huber, Joel, John W. Payne, and Christopher Puto (1982), “Adding Asymmetrically Dominated Alternatives: Violations of Regularity and the Similarity Hypothesis," Journal of Consumer Research, 18 (December), 325-45.

Huber, Joel and Christopher Puto (1983), "Market Boundaries and 
Product Choice: Illustrating Attraction and Substitution Effects," Journal of Consumer Research, 10 (June), 31-44.

Kahneman, Daniel, Jack L. Knetsch, and Richard H. Thaler (1991), "The Endowment Effect, Loss Aversion, and Status Quo Bias," Journal of Economic Perspectives, 5 (December), 1325-47.

Kassarjian, Harold (1974), "Projective Methods," in Handbook of Marketing Research, ed. Robert Ferber, New York: McGrawHill, 85-100.

Kivetz, Ran, Oded Netzer, and V. Srinivasan (2004), "Alternative Models for Capturing the Compromise Effect," Journal of Marketing Research 41 (August).

Luce, R. Duncan (1959), Individual Choice Behavior: A Theoretical Analysis. New York: Wiley.

Lynch, John G., Jr., and Dan Ariely (2000), "Wine Online: Search Costs Affect Competition on Price, Quality, and Distribution," Marketing Science, 19 (1), 83-103.

Nowlis, Stephen M. and Itamar Simonson (1996), "The Effect of New Product Features on Brand Choice," Journal of Marketing Research, 33 (February), 36-46.

Prelec, Drazen and George Loewenstein (1998), "The Red and the Black: Mental Accounting of Savings and Debt," Marketing Science, 17 (1), 4-28.

Quinlan, Philip T. and Richard N. Wilton (1998), "Grouping by Proximity or Similarity? Competition between the Gestalt Principles in Vision," Perception, 27 (4), 417-30.

Ratneshwar, Srinivasan, Allan D. Shocker, and David W. Stewart (1987), "Toward Understanding the Attraction Effect: The Implications of Product Stimulus Meaningfulness and Fa- miliarity," Journal of Consumer Research, 13 (March), 520-33.

Shepard, Roger N. (1962), "The Analysis of Proximities: Multidimensional Scaling with an Unknown Distance Function. Part I," Psychometrika, 27 (2), 125-40.

Simonson, Itamar (1989), "Choice Based on Reasons: The Case of Attraction and Compromise Effects," Journal of Consumer Research, 16 (September), 158-74.

Simonson, Itamar and Amos Tversky (1992), "Choice in Context: Tradeoff Contrast and Extremeness Aversion," Journal of Marketing Research, 29 (August), 281-95.

Tversky, Amos (1972), "Elimination by Aspects: A Theory of Choice," Psychological Review, 79 (October), 281-99.

(1977), "Features of Similarity," Psychological Review, 84 (4), 327-52.

Tversky, Amos and Daniel Kahneman (1991), "Loss Aversion in Riskless Choice: A Reference-Dependent Model," Quarterly Journal of Economics, 106 (November), 1039-61.

Tversky, Amos, Shmuel Sattath, and Paul Slovic (1988), "Contingent Weighting in Judgment and Choice," Psychological Review, 95 (July), 371-84.

Tversky, Amos and Itamar Simonson (1993), "Context-Dependent Preferences," Management Science, 39 (October), 1179-89.

Wernerfelt, Birger (1995), "A Rational Reconstruction of the Compromise Effect: Using Market Data to Infer Utilities," Journal of Consumer Research, 21 (March), 627-33.

Wertheimer, M. ([1923] 1999), "Laws of Organization in Perceptual Forms," in A Source Book of Gestalt Psychology, ed. W. D. Ellis, New York: Routledge, 71-88. 